\title{
DIE WAHRHEIT DES RELATIVEN IN DER KRISE DER FAKE NEWS
}

\author{
Denken mit Alexandra Juhasz' «\#100hardtruths \\ \#fakenews: A primer on digital media literacy»
}

\section{Eine Ökologie der Praktiken}

In ihren «Introductory Notes on an Ecology of Practices» geht Isabelle Stengers von Brian Massumis Vorschlag aus, dass «eine politische Ökologie eine soziale Technologie des Dazugehörens sei und dabei Koexistenz und Co-Becoming als das Habitat von Praktiken betrachte. $>^{1}$ Mit dieser Formulierung hatte Massumi im August 2003 zu einem Symposium ans Humanities Research Centre der Australian National University eingeladen. Stengers nimmt den Vorschlag auf, gibt ihm aber eine neue Wendung. Anders als Massumi legt sie die Ökologie der Praxis nicht als soziale Technologie, sondern als ein Werkzeug des Denkens aus. Eine Ökologie der Praxis wäre nach Stengers ein Werkzeug, das uns hilft, Gewohnheiten des Denkens zu ändern und über diese Änderung von Denkgewohnheiten den Praktiken zugleich ein neues Habitat bereitzustellen. «Ich verstehe», so definiert sie ihre Position, «unter einer Ökologie der Praxis ein Werkzeug, um gründlich durchzudenken, was aktuell geschieht, und ein Werkzeug ist niemals neutral». ${ }^{2}$

Stengers legt die Ökologie der Praxis als ein Problem des Denkens und damit verbunden als ein methodologisches Problem aus. Allerdings geht es ihr weder um Objektivität noch um Verallgemeinerbarkeit oder universale Wahrheit. Als Wissenschaftsphilosophin, die sich mit Fragen der Geschichtlichkeit von wissenschaftlichen Erkenntnissen beschäftigt, geht es ihr stattdessen um die Frage, wie Habitate von Praktiken sich verändern und wie Habitate verändert werden können.

Ausgehend von Stengers methodologischer Bestimmung des Denkens als eine Praxis, die eine Relation zwischen Gehören-zu (belonging) und Werden (becoming) stiftet, möchte ich im Folgenden zeigen, dass und wie eine so verstandene Ökologie der Praktiken einen Ausweg aus dem Diskurs der sogenannten
1 Isabelle Stengers: Introductory Notes on an Ecology of Practices, in: Cultural Studies Review, Vol. 11, Nr. 1, 2005, dx.doi.org/10.5130/csr. v11i1.3459, $183-196$, hier 183, Übers. Astrid Deuber-Mankowksy (AD).

2 Ebd., 185. Stengers benutzt zumeist den Plural «Ökologien der Praktiken", an dieser Stelle jedoch benutzt sie den Singular, «eine Ökologie der Praxis»: «What I call an ecology of practice is a tool for thinking through what is happening, and a tool is never neutral." 
3 Vgl. Wendy Hui Kyong Chun: Queerying Homophily: Muster der Netzwerkanalyse, in Zeitschrift für Medienwissenschaft, Nr. 18, 2018, 131-148.

4 Vgl. ebd.

5 Gilles Deleuze rechnete mit dieser kantischen Vorstellung der Vernunft als einer Richterin in seinem kurzen Text mit dem Titel "Schluss mit dem Gericht» ab. Vgl. ders.: Kritik und Klinik, Frankfurt/M. 2000, 171-183.

6 Vgl. Stengers: Introductory Notes, 185.

$7 \mathrm{Vgl}$. Ilya Prigogine [in collaboration with Isabelle Stengers]: The End of Certainty: Time, Chaos, and the New Laws of Nature, New York 1996.
Fake News weisen könnte. Was dies ganz konkret, also praktisch für die Frage heißt, wie unter Bezugnahme auf diesen methodologischen Ansatz mit dem Internet gedacht werden könnte, werde ich im zweiten Teil am Beispiel des digitalen Projektes \#100hardtrutbs-\#fakenews von Alexandra Juhasz vorstellen. Ein Fokus wird dabei auf dem Verhältnis von Habitat und der Änderung von Denkgewohnheiten liegen, das heißt zugleich auf dem Prozess der Transformation zwischen Gehören-zu (belonging) und Werden (becoming). Denn Denken mit dem Internet bedeutet Habitate zu schaffen, die offen sind für neue Muster und neue Formen von Relationen. ${ }^{3}$ Sie stellen sich gegen den Strom der Bubble-Bildungen und der perfomativen Prozesse, mit denen bestehende Diskriminierungen und Segregationen, wie Wendy Hui Kyong Chun in ihrer kritischen Analyse der Netzwerkforschung so eindringlich darlegte, wiederaufgeführt und naturalisiert werden. ${ }^{4}$

Wenn Stengers die Ökologie der Praxis als ein Denkwerkzeug bezeichnet, verweist sie darauf, dass Denken eine praktische Tätigkeit ist. Das meint, dass Denken Effekte zeitigt und nicht losgelöst ist von der Situation, in der es stattfindet. Mit der Betonung, dass ein Werkzeug niemals neutral sei, wendet sie sich gegen die Vorstellung, es handle sich hier um eine Methode, in der das Denken, wie es etwa Kant beschrieben hatte, nach Art eines Richters als urteilende Vernunft auftritt, die über der Situation steht, um festzustellen, um welche Situation oder eben um welchen Fakt es sich handelt. ${ }^{5}$ Ein Werkzeug ist in die Situation involviert, in der es zur Anwendung kommt, es trägt zur Bearbeitung und damit zur Veränderung dieser Situation bei. Zwischen dem Werkzeug und der konkreten Situation besteht, wie Stengers es ausdrückt, eine Beziehung von Relevanz. Relevanz meint, dass die Werkzeuge der Situation die Macht verleihen, uns zum Denken zu bringen. ${ }^{6}$

Die Verbindung von Gewohnheit und Habitat liegt im Englischen näher als im Deutschen, da babit - das englische Wort für Gewohnheit - etymologisch mit dem lateinischen habitat verbunden ist. Allerdings leuchtet die Verbindung auch im Deutschen ein, wenn man bedenkt, dass ein Habitat auch als Wohnung oder Umfeld des Wohnens verstanden werden kann. Habit und babitation lassen sich im Anschluss an Walter Benjamin als «Wohnen und Gewohnheit» übersetzen. Eine Änderung einer Gewohnheit führt entsprechend zu einer Änderung des Habitats.

Anders als Massumi bezieht Stengers ihre einführenden Bemerkungen zweitens auf eine bestimmte historische Wissenskonstellation, mit der sie als Philosophin und Epistemologin gut vertraut ist - lange hatte sie mit dem seinerseits unkonventionellen Physiker und Nobelpreisträger Ilya Prigogine über eine neue Interpretation des zweiten Hauptsatzes der Thermodynamik und über selbstorganisierende Systeme diskutiert und nachgedacht, um den physikalischen Praktiken des 20. Jahrhunderts so ein neues Habitat zu schaffen: ${ }^{7}$ auf das Verhältnis von Philosophie und Physik, oder genauer, auf das Verhältnis eines philosophischen Denkens, das sich an der Methodologie 
einer Ökologie der Praktiken orientiert, und einer Physik, deren Verständnis von physischer Realität, wie Stengers moniert, sich gegen die historischen Veränderungen immunisiert und immer noch auf den theologisch-politischen Forderungen des i 7. Jahrhunderts und damit der Gründungs- und Entstehungszeit der Physik als moderner Wissenschaft basiert. Mit der Definition der physikalischen Realität als objektive und jenseits der menschlichen Vorstellungen bestehende Realität beanspruche die Physik, wie Stengers kritisch zu bedenken gibt, eine exklusive Position des Urteils über und gegen alle anderen Realitäten, eingeschlossen die Realitäten von anderen wissenschaftlichen Disziplinen, und damit zugleich ein exklusives Recht auf Rationalität. Vergessen wir nicht: Dieses exklusive Recht auf Rationalität weist auf den Rationalismus des I7. Jahrhunderts zurück. Dessen jubilatorischer Glaube an die unbedingte Potenz der Rationalität aber hatte seinen Grund in der Überzeugung, dass die Existenz Gottes selbst rational beweisbar sei und dass die Rationalität andererseits ihre Beweiskraft ihrem göttlichen Ursprung verdanke. Eben diese Konjunktion von Rationalität, Gott und Gottesbeweis wurde bereits von Kant als dogmatisch erkannt und führte ihn zu seiner Reformulierung der Metaphysik als einer «Wissenschaft von den Grenzen der menschlichen Vernunft». ${ }^{\mathbf{8}}$

Stengers schrieb ihren Aufsatz vor dem Hintergrund der Science Wars, die 2002 noch lange nicht zu Ende waren. Der Krieg der Wissenschaften fand einen Höhepunkt in der Polemik des US-amerikanischen Physikers Alan Sokal. Sokal ist ein bekennender Vertreter des wissenschaftlichen Realismus, das heißt, er geht, ähnlich wie in jüngster Zeit die Vertreter_innen des spekulativen Realismus ${ }^{9}$ wieder, von der vorkantischen Annahme aus, dass eine erkennbare Wirklichkeit existiere, die unabhängig vom Denken und von der Geschichte sei und die wissenschaftlich beschrieben und repräsentiert werden könne. I 996 löste Sokal mit der Veröffentlichung eines Fake-Artikels in der Zeitschrift Social Text, den er später als Hoax outete, einen regelrechten Skandal aus. Sokal hatte in diesem Artikel mit dem Titel «Transgressing the Boundaries: Towards a Transformative Hermeneutics of Quantum Gravity» behauptet, bei der Quantengravitation handle es sich um eine soziale und linguistische Konstruktion. ${ }^{10}$ Ein Jahr später veröffentlichte Sokal zusammen mit Jean Bricmont einen Band, in dem die beiden Physiker unter dem Titel Fashionable Nonsens. Postmodern Intellectuals'Abuse of Science ${ }^{11}$ in Fortsetzung der Science Wars Stellen aus Texten von postmodernen Autor_innen einer kritischen Lektüre unterzogen und ihnen schlechte Wissenschaft vorwarfen.

Stengers legt diese Kriegserklärung der beiden Physiker als eine verständliche Reaktion gegen eine gefühlte Denunziation ihrer physikalischen Praxis aus. Wer wie die Vertreter_innen eines radikalen Konstruktivismus behaupte, Elektronen seien soziale Konstruktionen, greife nicht nur physikalische Glaubenssätze an, sondern denunziere auch jenes attachment, das die Physiker_innen mit den Elektronen verbindet, eben weil es sie zum Denken bringe
8 Immanuel Kant: Träume eines Geistersehers erläutert durch die Träume der Metaphysik, in: ders.: Werkausgabe, Bd. 2, Frankfurt/M. 1974, 918-989, hier 983.

9 Vgl. Quentin Meillassoux: Nach der Endlichkeit: Versuch über die Notwendigkeit der Kontingenz, Berlin, Zürich 2008; Maurizio Ferraris: Manifest des neuen Realismus, Frankfurt/M. 2014; Markus Gabriel (Hg.): Der neue Realismus, Berlin 2014.

$10 \mathrm{Vgl}$. Alan Sokal: Transgressing the Boundaries: Toward a Transformative Hermeneutics of Quantum Gravity, in: Social Text, Nr. 46/47, Spring/Summer 1996, 217-252.

$11 \mathrm{Vgl}$. Alan Sokal, Jean Bricmont: Fashionable Nonsens. Postmodern Intellectuals' Abuse of Science, New York 1999. 
12 Vgl. Stengers: Introductory Notes, 191. Stengers' Kritik richtet sich zentral an Bruno Latours und Steve Woolgars Laboratory Life. The Construction of Scientific Facts, Beverly Hills 1979. Die fehlende Anerkennung der wissenschaftlichen Praxis durch Latour kritisierte bereits Donna Haraway in der Einleitung zu Primate Visions. Gender, Race, and Nature in the World of Modern Science, New York, London 1989, 6.

13 Zur Dynamik dieses Verhältnisses vgl. Martin Doll: Fälschung und Fake: Zur diskurskritischen Dimension des Täuschens, Berlin 2012.

$\mathbf{1 4}$ Vgl. Stengers: Introductory Notes, 195.

15 Vgl. ebd., 196.

16 Karin Harrasser, Katrin Solhdju: Wirksamkeit verpflichtet. Herausforderungen einer Ökologie der Praktiken, in: Zeitschrift für Medienwissenschaft, Nr. 14, 2016, 72-86, hier 74 . und kreativ sein lasse. ${ }^{12}$ Sie zieht daraus den Schluss, dass es nicht darum gehen kann, dem Fake mit der Richtigstellung von Fakten zu begegnen. ${ }^{13}$ Denn damit würde man allzu leicht nur selbst auf den Rationalismus und die politischtheologischen Begriffe des I 7. Jahrhunderts mit den geschilderten Voraussetzungen zurückgreifen. Vielmehr gelte es, die Praktiken - hier die physikalische Praxis und den Umgang mit den Elektronen mitsamt dem attachment, das die Physiker_innen mit dieser Praxis verbinde - ernst zu nehmen. Die Aufgabe wäre, von diesen konkreten Praktiken auszugehen und ihnen ein neues Habitat zu geben, eines das der historischen Situation besser entspricht als die politisch-theologische Vorstellung der physikalischen Realität und Rationalität des I 7. Jahrhunderts.

In, um sie selbst noch einmal zu zitieren, «gründlich durchgedachter» Weise führt uns Stengers in ihrem Text von der Beobachtung, dass sich die zeitgenössische Physik in ihrem Selbst- und Realitätsverständnis immer noch im I 7. Jahrhundert befinde und als eine Praxis dringend eines neuen historischen Habitats bedürfe, zu einem Denken, das sie mit Deleuze als Denken «par le milieu» bezeichnet und das sie zum Schluss ihres Textes als eine Praxis beschreibt, die in gewisser Weise Analogien zu der Kunst der Magie aufweise. ${ }^{14}$ Ebenso wie jene stütze sich das Denken durch und mit dem Milieu nicht auf eine repräsentative, universale Wahrheit, sondern entfalte stattdessen eine transformative, eine verändernde Kraft, die sich auf eine bestimmte Situation bezieht. Wenn Stengers sich an dieser Stelle auf die Tradition der Hexenkunst beruft, kündigt sie zum einen die Komplizenschaft und das Einverständnis auf, mit dem ihre eigene Disziplin, die Philosophie, ihre Denkwerkzeuge im Namen der Universalität an die Physik abgegeben habe. Zum anderen situiert sie sich als Philosophin, die, wie sie schreibt, zwar einerseits mit den Denkwerkzeugen ihrer Disziplin denke, andererseits jedoch als Tochter dieser Disziplin mit jenen starken Frauen denke, die im Namen der Rationalität verfolgt wurden. ${ }^{15}$ Stengers praktiziert und führt uns damit durch jenes Denken, das durch den Bezug auf das aktuell Sich-Ereignende eine Relation zwischen Gehören-zu (belonging) und Werden (becoming) aktiviert.

\section{Denkgewohnheiten widerstehen}

Tatsächlich ist diese Verbindung von belonging, das als Gehören-zu in das aus der Geschichte Überlieferte führt, und becoming, dem als Werden eine verändernde Kraft in Hinblick auf das Zukünftige eignet, entscheidend für jenes Denken, das sich beim «gründlichen Durchdenken» dessen, was im Geschehen begriffen ist, von alten Denkgewohnheiten löst. Karin Harrasser und Katrin Solhdju haben diese Forderung in ihrer Darstellung der Herausforderungen einer Ökologie der Praktiken auf die Frage zugespitzt: «Kurz, wie lässt sich das Material so angehen, dass es zu einer Gelegenheit für die Schärfung und Transformation des eigenen Denkens wird? ${ }^{16}$ 
Stengers nennt es ein Denken in minor keys und stellt es dem Denken in major keys gegenüber, das eine Konvergenz zwischen Freiheit und Wahrheit unterstelle. Stellt, so möchte ich fragen, eine Praxis des Denkens, das eine Relation zwischen Gehören-zu (belonging) und Werden (becoming) stiftet, nicht eben jene Grundlagen bereit, welcher ein kritisches Denken jenseits der Ideologiekritik unter den heutigen, aktuellen technischen, ökonomischen und politischen Bedingungen des Internets bedarf? Das Internet, in dem und mit dem wir heute einen bedeutsamen Teil unseres Alltags verbringen, hat nach der Transformation in das Web 2.0 seit ungefähr 2004 die Form eines auf dem Konzept des neoliberalen Geschäftsmodells ${ }^{17}$ basierenden Social Web angenommen. Dazu gehören die spezifische Geschichte seiner Infrastruktur, seiner Algorithmen, seiner Plattformen und seiner Kommerzialisierung sowie die zunehmende Vereinnahmung der offenen Netzstruktur des Internets in die ökonomische und staatliche Überwachungs- und Regulierungspolititik. Als Social Web stellt das Internet das kritische Denken vor eine besonders harte Bewährungsprobe. Wie ist es möglich, so könnte man diese Herausforderung umschreiben, unter den Bedingungen der Neoliberalisierung, Ökonomisierung und Überwachung mit all den gewaltsamen Folgen sogenannter Fake News, des Rassismus, der Homophobie und Mysogynie im Internet als Teil unserer Umwelt, ein Habitat zu schaffen, in dem Zugehören und Werden sich in dem oben beschriebenen Sinn aufeinander beziehen? Stengers legt die transformative Kraft des Denkens, die sich in der spannungsvollen Relation von Zugehören und Werden ereignet, als eine Befähigung (empowerment) aus. Und es ist, wie ich im Folgenden an dem Beispiel von Alexandra Juhasz' «\# Ioohardtruths-\#fakenews: A primer on digital media literacy. Generating principles for each deceptive day, January 20 - April 29, 201 $7 \gg^{18}$ zeigen möchte, genau diese Form der Befähigung, die auch ein (kritisches) Denken mit dem Internet auszeichnet.

Wenn ich das Denken, das Stengers als Denken in minor keys bezeichnet, mit der Herausforderung des aktuellen kritischen Denkens in Beziehung setze, schließe ich mich Judith Butler an, die in ihrer Erinnerung an die Lektüre von Alfred North Whiteheads Prozessphilosophie und in deren Relektüre in Anknüpfung an Stengers davon ausgeht, dass kritisches Denken mehr aufzeigt, als es sagt, und dass es tiefer in ein Netz von Beziehungen eingelassen ist, als es selbst sagen und wissen kann. ${ }^{19}$ Damit aber fällt kritisches Denken gerade nicht mit jenem Denken zusammen, das Stengers als Denken in major keys definiert und das, wie sie schreibt, Freiheit an eine Wahrheit knüpft, die als absolut und repräsentativ auftritt. ${ }^{20}$ Gegen diese Verbindung von Macht und Wahrheit setzt Stengers die «critique <par le milieu»», ${ }^{21}$ die sich an einem pragmatischen Ethos orientiert, die eine situationsgebundene Entscheidung sucht und keine Kollateralschäden in Kauf nimmt. Stengers spricht in diesem Zusammenhang von einer Technologie, die sich statt an der Macht der Wahrheit an Testerfabrungen $^{22}$ orientiert.
17 Bereits im Titel des wegweisenden Artikels, in dem O'Reilly 2004 den Begriff des Web 2.0 einführte und in einer Art Manifest zusammenfasste, was das Web 2.0 gewesen sein wird, taucht «Geschäftsmodell? als die zentrale Form des neuen Web auf. Vgl. Tim O'Reilly: What Is Web 2.o. Design Patterns and Business Models for the Next Generation of Software, dort datiert 30.9.2005, online unter oreilly.com/pub/a/web2/ archive/what-is-web-20.html, gesehen am 20.1.2018.

18 Vgl. Alexandra Juhasz: \#10ohardtruths-\#fakenews: A primer on digital media literacy. Generating Principles for each deceptive day, January 20 - April 29, scalar.usc.edu| nehvectors/10ohardtruths-fakenews/ index, gesehen am 20.1.2018.

19 Vgl. Judith Butler: On this Occasion, in: Roland Faber, Michael Halewood, Deena Lin (Hg.): Butler on Whitehead: On the Occasion, Plymouth 2012, 3-19, hier 3. Butler knüpft hier an Stengers Aussage in ihrer Whitehead-Interpretation an: «critical consciousness admits so many things without criticizing them".

$20 \mathrm{Vgl}$. Stengers: Introductory Notes, 187.

21 Ebd.

22 Vgl. ebd., 188. 
Ein wichtiger Berührungspunkt zwischen den Anliegen kritischen Denkens und dem Denken in minor keys liegt dort, wo Stengers aus einer «Ökologie der Praxis als Werkzeug für das Denken» die Aufforderung ableitet, Denkgewohnheiten zu widerstehen. Um (Denk-)Gewohnheiten zu widerstehen, müssen wir in einer Situation jedoch zunächst anstoßen. Wir müssen, wie Stengers formuliert, die Potenzialität wahrnehmen, die uns zum Denken bringt. ${ }^{23}$ Diese Potenzialität bedarf der Aktualisierung, damit das Denken seine transformative Kraft entfalten kann. Die relevanten Werkzeuge des Denkens, die Stengers aus einer Ökologie der Praxis gewinnt, sind eben jene, welche es uns erlauben, eine Situation für uns so bedeutsam zu machen, dass sie uns zu denken gibt. An einer Stelle verweist Stengers zu dessen genauerer Charakterisierung auf die Praxis der Experimentalphysiker_ innen: So wie die Physiker_innen in ihren Versuchsanordnungen die Dinge aus dem Gleichgewicht bringen, stellt uns, so Stengers, eine Ökologie der Praktiken außerhalb unserer Gewohnheiten und erlaubt uns auf diese Weise, ein verändertes Habitat zu schaffen, das den historischen Gegebenheiten angemessen ist. ${ }^{24}$

$\mathrm{Zu}$ diesen historischen Gegebenheiten gehören heute die bereits beschriebenen technischen, ökonomischen, politischen Bedingungen des Internets mit all den dazugehörigen Praktiken. Wie ließe sich in diesem so korrumpiert erscheinenden Internet und für dieses ein neues Habitat schaffen? Ich werde im Folgenden Alexandra Juhasz' Projekt \#100hardtruths-\#fakenews als eine möglich Antwort auf diese Frage vorstellen. Juhasz begegnet dem Diskurs der sogenannten «fakenews» nicht mit der Gegenüberstellung von Fakten, sondern ergänzt sie vielmehr mit «hardtruths», mit bitteren Wahrheiten, und versucht, die Widersprüche des Internets mit den technischen und sozialen Praktiken des Internets selbst zu denken.

\section{\#100hardtruths-\#fakenews}

Am 8. Dezember 2016 hatte Donald Trump die Präsidentschaftswahlen in den Vereinigten Staaten gewonnen, am 20. Januar 2017 fand seine Inauguration statt. Um die völlig willkürlich gefälschte Angabe über die Zahl der anwesenden Zuschauer_innen zu verteidigen, erfand Kellyanne Conway, die Sprecherin der neuen Regierung, die Formulierung der «alternativen Fakten». Dies stellte jedoch nur eine Zuspitzung der Fake-News-Diskurse und der gezielten Manipulationen dar, die bereits im Vorfeld der Wahlen über und mithilfe des Internets deren Ausgang zentral beeinflusst hatten.

Alexandra Juhasz, seit 20 I6 Direktorin des Film Departments am Brooklyn College in New York, reagierte auf diese Situation mit dem Projekt \#100hardtruths-\#fakenews. Die ıoo Tage vom 20. Januar bis zum 29. April waren die ersten Ioo Regierungstage des neuen Präsidenten und umfassen die Frist, die

23 Vgl. Stengers: Introductory Notes, 185 .

24 Vgl. ebd., 195. dem neuen Amtsinhaber traditionell zugestanden wird, um sich einzuarbeiten und erste Erfolge vorzuweisen. Nach diesen roo Tagen kommt es normalerweise zu einer ersten Bilanz. 
Alexandra Juhasz betraf diese Situation ganz direkt als Wissenschaftlerin und Aktivistin, die seit ihrer Zeit als Studentin Medienaktivismus und medienwissenschaftliche Analyse miteinander verbunden hatte. Mit Stengers könnte man sagen, dass die von Trump und seinen Wahlhelfer_innen lancierten Fake-News-Diskurse das attachment berührten und verletzten, das Juhasz mit dem Medium verband, in dem und mit dem sie forschte. Dieses attachment beruht auf einer fast 30-jährigen Forschungspraxis, die zusammen mit dem in dieser Zeit entstandenen Netzwerk und den medialen Projekten in das \#100hardtruths\#fakenews eingeflossen ist und einen wichtigen Teil seines Inhaltes bildet:

Juhasz hatte in den späten I980er Jahren - während der Zeit der ACTUP-Bewegung - in New York Filmwissenschaft studiert und das Studium I 992 mit einer Dissertation zum Thema «Re-Mediating AIDS: The Politics of Community Produced Video» an der NYU abgeschlossen. Sie war selbst ein aktives Mitglied der ACT-UP-Bewegung und verbindet seit jener Zeit in ihrer Forschung Medienproduktion, -praxis und -wissenschaft. Sie unterrichtete von 1995 bis 2016 am Pitzer College als Professorin und Direktorin des Monroe Center for Social Inquiry. Ihre Forschungsinteressen gelten insbesondere dem Genre des Dokumentarischen im Feld der feministischen Filmwissenschaft und Queertheorie. Sie produzierte die Spielfilme The Watermelon Woman (Cheryl Dunye, USA I996) und The Owls (Cheryl Dunye, USA 2010) und mehr als ein Dutzend Dokumentarfilme für Unterrichtszwecke über Themen wie Minderjährigenschwangerschaften und AIDS. The Watermelon Woman war der erste Film über die (nicht existente) Geschichte von afroamerikanischen lesbischen Schauspielerinnen in der US-amerikanischen Filmindustrie. ${ }^{25}$ Zusammen mit der Medienwissenschaftlerin Anne Balsamo gründete Juhasz 2012 das feministische Netzwerk FemTechNet, das sich als «new approach to a collaborative learning» und als ein «activated network of scholars, artists and students» versteht, «das über, mit und an den Grenzen zwischen Technologie, Wissenschaft und Feminismus in einem interdisziplinären Feld arbeitet, das STS, Medienwissenschaft und Visual Studies, Kunst, Gender- und Queerstudies und Ethnologie verbindet.» ${ }^{\mathbf{2 6}}$ Wie durch ihre zahlreichen Publikationen in diesem Bereich deutlich wird, ist sie eng vertraut mit den Fallstricken und Potenzialen des Dokumentarischen und des Dokumentierens. ${ }^{27}$ Juhasz reagierte weder mit einer Apologie des <wahren> Internets noch mit einer enttäuschten Abkehr und Verwerfung auf diese Situation, sondern sie entschied sich, auf neue Weise im Internet über das Internet nachzudenken. Man kann dies als das Herstellen einer Beziehung von Relevanz verstehen, als eine Aktualisierung der Potenzialität, die dieser konkreten Situation innewohnt. Am i 8. Februar, drei Wochen nach der Inauguration von Donald Trump, formulierte Alexandra Juhasz in ihrem Blog zwei Selbstverpflichtungen, die in der Folge wie selbstauferlegte Regeln eines Spiels fungierten. Sie eröffnete damit das testende Vorgehen, mit dem sie in der Folge ein neues Habitat im Internet kreieren sollte:
25 Siehe dazu auch den Beitrag von Andreas Sudmann in diesem Schwerpunkt, insbesondere $47 \mathrm{f}$.

26 FemTechNet: About, femtechnet. org/about|, gesehen am 20.1.2018, Übers. AD.

27 Vgl. Alexandra Juhasz, Jesse Lerner: $F$ is for Phony. Fake Documentary and Truth's Undoing, Minneapolis 2006; dies.: Learning from YouTube, Cambridge 2011, online unter vectors. usc.edu/projects/learningfromyoutube/, gesehen am 10.7.2018; dies., Alisa Lebow (Hg.): Blackwell Companion to Film Studies: Documentary and Documentary Histories, Cambridge 2016. 
I. Sie werde die ersten ıoo Tage der Präsidentschaft von Trump durch das Posten von \#100hardtrutbs-\#fakenews und damit verbundenen Aktionen, Analysen und Organisationen unterbrechen, die alle der Förderung der digital media literacy - der Förderung der digitalen Medienkompetenz - gewidmet sein sollen.

2. Indem sie dies tue, werde sie eine roo Punkte umfassende Fibel für den Umgang mit digitalen Medien produzieren, um damit der Verwirrung, dem Vertrauensverlust und der Desorientierung der aktuellen Regierung im Umgang mit Medien entgegenzuwirken, und stattdessen ein verlässliches und durchdachtes Set von Quellen und Hilfsmitteln zur Verfügung stellen, die sich an Transparenz, Klarheit und Gerechtigkeit orientieren. ${ }^{28}$

Die ersten 50 Posts veröffentlichte sie in ihrem Blog, auf Facebook und auf Twitter, um ein möglichst großes Publikum zu erreichen, das sie zugleich zur aktiven Beteiligung an dem Projekt einlud.

Die Absicht war, an die Internetpraktiken anzuknüpfen und mit den medialen und ästhetischen Formen des Internets zu denken. Dazu gehört nicht nur, dass Juhasz sich des Bloggens und der medialen Plattformen Facebook und Twitter bediente, sondern auch, dass sie der zeitlichen Ordnung der vernetzten Zeit folgte. Diese Zeitordnung zeichnet sich, wie Isabell Otto überzeugend argumentiert, durch eine Prozesshaftigkeit aus, die sich ihrerseits aus der Gesamtheit der Praktiken des Zeitordnens ergibt. ${ }^{29}$ Dieser Prozess ist relational, das heißt, er generiert seine Ordnung nicht durch den Bezug auf eine unabhängige und absolute Zeitgröße, sondern aus der Summe all der miteinander vernetzten und sich aufeinander beziehenden Praktiken und Aktivitäten. ${ }^{30}$ Mit ihrem Projekt klinkte sich Juhasz in diesen Prozess ein. Das heißt, im Fluss der roo Tage roo vernetzte, aphoristische, auf andere Seiten und Projekte verweisende und verlinkende Analysen zu produzieren. Dabei richtete sich ihre Aufmerksamkeit zugleich auf die Architektur des Internets, seine technische und ökonomische Infrastruktur, auf die vorliegende Literatur der kritischen Internetstudien, auf Möglichkeiten des Widerstands und die täglich sich ändernden Fake-Behauptungen, mit denen die Trump-Regierung die aktuelle Situation auf gefährliche und zerstörerische Weise zuspitzte.

Juhasz erster Blogeintrag beginnt am I 8. Februar 2017 mit einer hardtruth, einer bitteren Wahrheit, über die Verfasstheit des Internets: Sie gipfelt darin, dass sich hinter der Frage nach der <Realität $>$ des Internets (Protokoll, Netz-

28 Alexandra Juhasz: My Pledge, in: dies.: \#10ohardtruths-\#fakenews, dort datiert 13.1.2018, scalar.usc. edu/nehvectors/10ohardtruths-fakenews/ about, gesehen am 20.1.2018, Übers. AD.

29 Vgl. Isabell Otto: Die Zeit der Vernetzung: Zeitordnungen unter der Bedingung digitaler Medien, unveröffentl. Habilitation, eingereicht an der Universität Konstanz 2017. $30 \mathrm{Vgl}$. ebd. werk, Regierungen, Wirtschaft, Technologie etc.?) ebenso wie nach dessen $\langle$ Fake $>$ (Illusion der Freiheit, der Demokratie, der Offenheit, der Selbstdarstellung etc.?) eine Komplexität verberge, die sich nicht so einfach in das Schema von <wahr> und <falsch> füge. Diese Komplexität sei, so schließt Juhasz, am besten über Aphorismen darstellbar, denn diese seien einerseits konzentriert und andererseits zugleich kurz und beweglich und ließen sich daher mit der Komplexität verknüpfen, die durch Forschung, Schreiben, Daten, Community und Kontext dazukomme. 
SOME \#10OHARDTRUTHS OF THE INTERNET (2/22)

\section{\#1: the real internet is a fake}

\section{and \\ \#7: skeptical interaction with the digital is critical for democracy}

\section{SOME \#10OHARDTRUTHS ON RACISM (1/19) \#20: stress related to immigration status is one result}

Zur weiteren Lektüre finden sich im gleichen Eintrag Links u.a. zu Alexander Galloway und Eugene Thackers Protocol, Control, and Networks und Wendy Hui Kyong Chuns On Software, or the Persistence of Visual Knowledge. Juhasz zieht daraus den Schluss, dass dem Internet und seinen News und Netzwerken nicht zu trauen ist, was wiederum bedeutet, dass eine skeptische und selbstbewusste Interaktion mit digitalen Daten die kritische Fundierung darstelle, auf der die Demokratie erhalten werden könne.

Wie bereits der Titel \#100hardtruths-\#fakenews anzeigt, geht Juhasz nicht von der Binarität von Wahrheit und Fake oder Realität und Virtualität aus, sondern davon, dass die Realität des Internets voller Widersprüche ist und widersprechende Aussagen zulässt. Die Aufgabe, die sich für das Projekt von Juhasz daraus ergibt, ist nicht, diese Widersprüche aufzulösen, sondern eine Antwort auf die Frage zu finden, wie im Internet mitsamt seinen Widersprüchen ein Habitat geschaffen werden kann, das der Komplexität unserer digitalen Realität gerecht wird. Das Projekt \#100hardtruths-\#fakenews erweist sich damit als ein experimentelles und testendes Vorgehen ganz in jenem Sinn, in dem Stengers das Denken in minor keys beschreibt und mit den Versuchsanordnungen der
Abb. 1-3 v. o. n. u. Blogeinträge vom 18.2., 19.2. und 25.2.2017 


\section{\#100hardtruths-\#fakenews}

A primer on digital media literacy

Generating principles for each deceptive day, January 20, 2017-April 29, 2017

Make sure to click through each hardtruth for ever more honesty, useful resources, and further connections

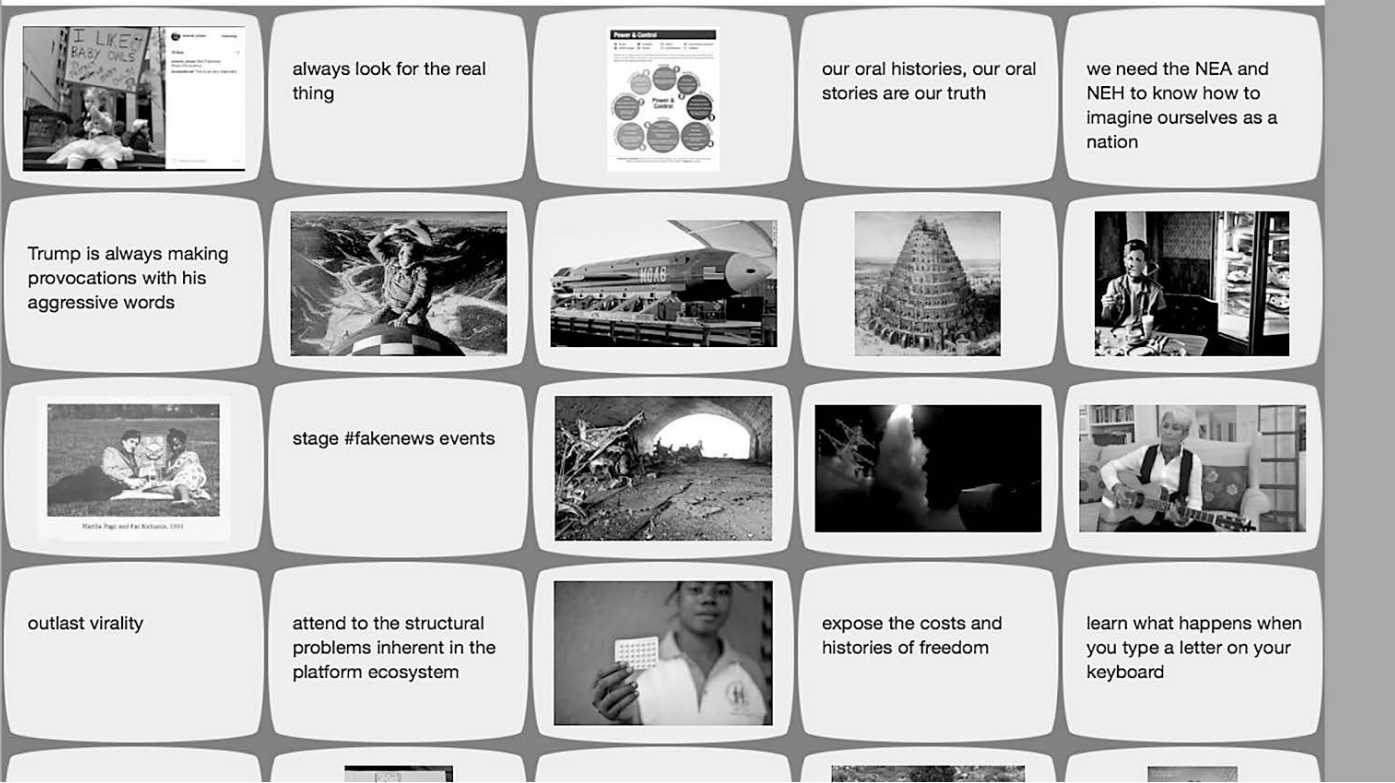

Abb. 4 Die Plattform \#100bardtrutbs-\#fakenews
Physiker_innen vergleicht. In ähnlicher Weise wie diese bringt Juhasz mit ihren selbstauferlegten Regeln die Interaktion mit digitalen Daten aus dem Gleichgewicht, stellt eigene Denkgewohnheiten auf den Prüfstand und versucht, die Situation im und mit den medialen Mitteln des Internets neu zu durchdenken. Juhasz beruft sich dabei auf ein existentes und weites Beziehungsnetz, dem sie sich zugehörig fühlt - von den kritischen Internetstudien über die Mitglieder des Netzwerks FemTechNet, Freund_innen, Familienmitglieder bis hin zu politischen Initiativen, die Informationen für Menschen zusammenstellen, die durch die Politik der Trump-Regierung in Gefahr sind. Aktuell betraf dies vor allem illegale Immigrant_innen, die seit Jahren und Jahrzehnten in den USA leben und arbeiten, Kinder mit US-amerikanischen Pässen haben und von Abschiebung bedroht sind. In verlinkten Videos werden die Rechte erklärt, auf die sie sich berufen können, und wird gezeigt, worauf sie bei drohenden polizeilichen Kontrollen achten sollten.

Mit jedem Tag und jedem Posting wuchs und verdichtete sich im ersten Monat ein Netz an Daten: Videos, Bilder, Texte, Verweise auf Quellen, Literatur, Tipps, Informationen, Reflexionen und Lernprozesse. In ihrem am I9. März 
veröffentlichten 50. Post zog Juhasz eine Konsequenz aus den Grenzen, an die sie mit ihrem Projekt stieß, und fügte zwei neue Selbstverpflichtungen (pledges), die ich hier mit Spielregeln übersetze, hinzu. ${ }^{31}$ Es zeigte sich, dass die Oberflächlichkeit und die Schnelllebigkeit der Kommunikation per Blog und das Teilen der Posts über die Plattformen Twitter und Facebook die transformative Kraft des Denkens nicht fördern, sondern behindern. Deren strukturelle Logik der neoliberalen Produktion, Konsumption und des Unternehmenseigentums, deren Wertschätzung des Schnellen, Oberflächlichen und Viralen und die Vorspiegelung von Gemeinschaft, Engagement und Teilnahme, welche nur die Erfahrung der Isolation, Zerstreuung und Kommodifizierung überdecken, passten nicht zu den Zielen und Werten, die aus dem Projekt selbst erwuchsen.

Juhasz entschied sich deshalb für den Aufbau einer eigenen Plattform, die eine andere Darstellung und eine andere Ausdrucksform ermöglichte. Beim Aufbau und Design dieses neuen Habitats wurde sie von Craig Dietrich unterstützt, Entwickler und Information Design Director der Alliance for Networking Visual Culture und deren Online-Plattform Scalar. ${ }^{32}$ Das nichtkommerzielle Netzwerk wurde mit finanzieller Unterstützung der Mellon Foundation aufgebaut und u.a. von Wendy Hui Kyong Chun, Nicholas Mirzoeff und Tara McPherson entworfen. Seine Finanzierung wurde mit dem Ziel eingeworben, eine freie, Open-Source-basierte Publikationsplattform zu entwickeln, die den Anforderungen der Darstellung von komplexen theoretischen und ästhetischen geistes- und kulturwissenschaftlichen Inhalten und Praktiken gerecht werden sollte. Der Name Craig Dietrich ist in den credits neben jenem von Alexandra Juhasz aufgeführt. Es bewahrheitete sich, anders formuliert, dass es nicht ausreicht, innerhalb der gegebenen kommerziellen Netzstrukturen und Plattformen aktiv zu werden, sondern dass die Technik und die Strukturen selbst angeeignet, um- und mitgebaut werden müssen.

Seit dem I9. März kann man sich von einer Homepage aus durch die als kleine Bildschirme simultan sichtbaren Posts klicken, den weiterführenden Links nachgehen und mit einem Klick zum Ausgang und zu der bis zum 29. April, dem roo. Tag von Trumps Regierungszeit, auf die Anzahl von Ioo Posts gewachsenen Website sowie zu den Spielregeln - den zwei anfänglichen und den in der Halbzeit ergänzten weiteren zwei pledges - zurückkehren. Mit den zwei neuen Regeln suchte Juhasz Offline-Prozesshaftigkeit in der Online-Realität stärker zum Zug zu bringen und damit die OnlineProzesse zum einen zu verlangsamen und ihnen zum anderen mehr Verbindlichkeit zu verleihen. Mit der ersten neuen Regel verpflichtete sich Juhasz, ihr Projekt - die kritische Unterbrechung der ersten roo Tage von Trumps Regierung - mit anderen zu teilen, indem sie andere bitten würde, eigene \# Ioohardtruths zu verfassen, um auf diese Weise anzuerkennen, dass das wichtigste und wertvollste Wissen von communities of practice and care komme. ${ }^{33}$ Die zweite Regel besagte, dass Juhasz für die restlichen \# 5 ohardtruths
31 Vgl. Alexandra Juhasz: 5 \#hardtruths and 2 new pledges (a) 50 , in: dies.: \#10ohardtruths\#fakenews, dort datiert 17.12.2017, scalar.usc.edu/nehvectors/100hardtruthsfakenews/5-hardtruths-and-2-newpledges-1, gesehen am 20.1.2018 $32 \mathrm{Vgl}$. The Alliance for Networking Culture: About The Alliance, scalar.me/anuc/about/, gesehen am 20.1.2018.

33 Vgl. Alexandra Juhasz: 5 \#hardtruths and 2 new pledges @ 50. 
Leute über eine One-to-one- und Person-to-person-Kommunikation (über Mail oder in real life) einladen würde, an dem Projekt teilzunehmen, um damit ihre eigenen Wahrheiten mit Wahrheiten von Leuten zu verbinden, die sie persönlich kennt.

In der Folge bereichern Perspektiven aus sehr unterschiedlichen Orten und Erfahrungen sowie Expert_innenwissen die wachsende Zahl an Verweisen auf affirmative Praktiken und Projekte, wie jene von Danielle Jackson, der Mitbegründerin des Bronx Documentary Center, Laura Wexler, Principal Investigator des von der National Endowment for the Humanities unterstützten Photogrammar Project, Eve Oishi, Kuratorin und Kulturwissenschaftlerin, und vielen anderen. Man kann in diesem neu geschaffenen digitalen Habitat Stunden, ja Tage verbringen. Man kann Neues lernen, etwa wie man Internetquellen zitiert und warum nicht alles, was online zugänglich ist, auch ungefragt zitiert werden sollte. ${ }^{34}$ Kurz: Man kann digital literacy erwerben. Es ist eine Versammlung von nachdenklichen und fragenden Stimmen, von digitalen und analogen Widerständen, die Juhasz in ihrem ıoo. Post mit dem bekannten Bild des japanischen Künstlers Hokusai Die große Welle (I829-I832) und dem begleitenden Zitat der Schriftstellerin Eileen Myles fasst: «We can't build a wall. We can only spout pure water again and again and drown his lies.»

Sie beendete die zweite neue Spielregel mit der Regel, dass sie alle Leute, mit denen sie ihr Projekt teilt, bitten würde, es ihrerseits mit Leuten zu teilen, die sie kennen. Ich habe von den \#100hardtruths-\#fakenews am I3. April 2017 erfahren, als Alexandra Juhasz es im Seminar Sites of Cinema an der Columbia University in New York vorstellte, und bin ihrer Regel und Aufforderung hiermit gefolgt. Nach Abschluss der ıoo Tage ergänzte Juhasz den Untertitel der Fibel für digitale Medienkompetenz mit einem Halbsatz, der jene transformative Kraft beschwört, die Stengers mit einer ecology of practices verbindet und welche freigesetzt wird, wenn es gelingt, belonging (Gehören-zu) mit becoming (Werden) zu verbinden, damit etwas Neues und eine Zukunft entstehen. Er lautet: «Make sure to click through each hardtruth for ever more honesty, useful resources and further connections»! Die Öffnung für neue Verbindungen ergänzt sich mit dem Schluss, den Wendy Hui Kyong Chun aus ihrer Analyse der rückwärtsgewandten Identitätspolitik in digitalen Netzwerken zieht, die durch die aktuelle Netzwerkforschung performativ verstärkt wird: «Die Zukunft liegt in den neuen Mustern, die wir gemeinsam kreieren können - neue Formen für Relationen, die lebbare Muster der Indif-

$34 \mathrm{Vgl}$. Alexandra Juhasz: \#93, citation ist not enough, in: dies.: \#10ohardtruths-\#fakenews, dort datiert 17.12.2017, http://scalar. usc.edu/nehvectors/10ohardtruthsfakenews/93-citation-is-not-enough, gesehen am 20.1.2018.

35 Chun: Queerying Homophily, 148. ferenz ermöglichen.» ${ }^{35}$

\section{Postscriptum}

Am I3. August 2017 erhielt ich eine E-Mail von Alexandra Juhasz, in der sie ankündigte, dass der nächste Schritt ihres Projektes «lecture-performance- 
pedagogic-manifestations» sein werden. Diese sollen situiert sein, das heißt orts- und communitygebunden, live und über einen Zeitlauf, so komplex und multidisziplinär wie das Problem selbst, offen für alle, die interessiert sind, fließend in den Sprachen der Theorie, Politik, des Affekts und der Kunst.

Die Mail enthielt eine Bitte um Einladung und auch eine Einladung zur Teilnahme, die eigenen bitteren Wahrheiten über das Internet zu aktivieren und sie kollaborativ in das Projekt einzubringen. Das Denken par le milieu im Internet und um das Internet geht also weiter und alle sind eingeladen, daran teilzunehmen. 